\title{
Spectral prediction model for piles of nonscattering sheets
}

\author{
Mathieu Hébert, ${ }^{1, *}$ Roger D. Hersch, ${ }^{1}$ and Lionel Simonot ${ }^{2}$ \\ ${ }^{1}$ Ecole Polytechnique Fédérale de Lausanne (EPFL), School for Computer and Communication Sciences, \\ 1015 Lausanne, Switzerland \\ ${ }^{2}$ PHYMAT (Laboratoire de PHYsique des MATériaux), UMR CNRS 6630, Boulevard Marie et Pierre Curie, \\ BP 179, 86962 Futuroscope Chasseneuil Cedex, France \\ *Corresponding author: mathieu.hebert@epfl.ch
}

Received March 21, 2008; revised June 13, 2008; accepted June 16, 2008; posted June 16, 2008 (Doc. ID 94143); published July 22, 2008

\begin{abstract}
The present paper investigates the reflection and transmission properties of piles of nonscattering sheets. Using a spectral prediction model, we perform a detailed analysis of the spectral and color variations induced by variations of the number of superposed sheets, the absorbance of the sheet material, the refractive index of the medium between the sheets, and the reflectance of the background. The spectral prediction model accounts for the multiple reflections and transmissions of light between the interfaces bounding the layers. We describe in detail the procedure for deducing model parameters from measured data. Tests performed with nonscattering plastic sheets demonstrate the excellent accuracy of the predictions. A large set of predicted spectra illustrate the different evolutions of reflected and transmitted spectra as well as the corresponding colors for various types of piles. (C) 2008 Optical Society of America
\end{abstract}

OCIS codes: $120.5700,120.7000,230.4170,300.6170,300.6550$.

\section{INTRODUCTION}

It is known that the color of an object is defined both by the intrinsic optical properties of matter and by the optical behavior of its surface. The surface reflectivity, which at the air side is responsible for the gloss effect, is also important within the interior of the object. A significant part of light is internally reflected and thus travels several times between the object's bulk and its surface before emerging. This phenomenon tends to amplify the coloration of light by a colored object. Accordingly, Saunderson [1] proposed a correction of the Kubelka-Munk reflectance model [2] accounting for this multiple reflection phenomenon. With respect to color images printed on paper, the models of Williams and Clapper for photographs [3], later revisited by Shore and Spoonhower [4], and the model of Clapper and Yule for halftone prints [5] explicitly embody the multiple reflections of light beneath the surface.

In the Williams-Clapper model, the coloring layer is a nonscattering gelatin deposited on top of a diffusing background (paper bulk). Even though the light issued from the background is diffuse, the different light rays propagate through the gelatin along straight lines and are attenuated according to their orientation. Once reaching the surface, they are internally reflected in proportions depending again on their orientation. The orientationdependent behavior of light within a nonscattering coating is one of the major contributions of Williams and Clapper. In contrast, mainly because the inks penetrate the paper support and form a diffuse coloring layer, the Clapper-Yule model ignores the orientation of light.

The Williams-Clapper model and the Clapper-Yule model may be qualified respectively as "orientational" and "nonorientational" reflectance models [6]. An orienta- tional model recently proposed by Simonot et al. [7] extends the Williams-Clapper model to specimens where the nonscattering coloring layer and the diffusing background have different refractive indices. Multiple reflections occur between the background and the coloring layer-background interface as well as between the coloring layer-background interface and the coloring layer-air interface. The multiple reflection process accounts explicitly for the orientation of light in the different media.

In the present paper, we extend the study of reflecting specimens composed of diffusing and transparent layers by considering piles of identical nonscattering sheets deposited on a diffusing support. We create a large number of specimens by varying the number of superposed sheets, the type of support, and the medium binding the different sheets and the support. With this large measured dataset, we verify the prediction model introduced by Simonot et $a l$. and study the influence of its underlying parameters. In Section 2, we recall basic notions of geometrical optics as well as reflectance and transmittance expressions for nonscattering layers bounded by flat interfaces. In Section 3 , we show how the normal transmittance of a nonscattering sheet is deduced from measurements. In Section 4 , we verify the applicability of the reflectance and transmittance prediction model of Simonot et al. and characterize the reflectance, transmittance, and color variations as a function of the medium between the sheets and of the number of superposed sheets. In Section 5 , we present a simpler model for the special case where the piles are separated one from another by air. Section 6 deals with the case where the piles are superposed onto a diffusing support. We develop the corresponding prediction model, verify it experimentally, and use it to describe 
spectral and color variations with respect to the influencing parameters. Our conclusions are given in Section 7.

\section{MODEL FOR STACKED NONSCATTERING LAYERS}

A nonscattering layer bordered by media with different refractive indices forms, together with its bounding interfaces, a reflective and transmissive element called an "interfaced layer." Light is reflected multiple times between the two interfaces and attenuated by absorption within the layer. Stacked nonscattering layers form an "interfaced multilayer." Reflectance and transmittance expressions for interfaced layers and interfaced multilayers have been developed by Simonot et al. [7]. They are recalled in the present section. We first present the assumptions, than the notations and the basic laws of geometrical optics relative to layers, interfaces, and measurement geometries.

\section{A. Assumptions}

Light is assumed to be incoherent and unpolarized. The nonscattering layers are infinitely large, homogenous, and isotropic, and their thickness is large compared to the wavelength of light. Interference phenomena are therefore neglected. The layer interfaces are flat. The interfaces, the interfaced layers, and the interfaced multilayers are placed horizontally; their two faces are called "upper" and "lower" sides. Their reflectance and transmittance, as well as the angles of reflection and of transmission, depend on the side and on the angle of incidence of light. Hence, they are characterized by an upper reflectance and a downward transmittance when illuminated from the upper side, and by a lower reflectance and an upward transmittance when illuminated from the lower side.

\section{B. Notations}

The refractive indices are noted $n_{i}$ and the light propagation angles $\theta_{i}$, where subscript $i$ is a labeling number for each medium. The interface between a medium $i$ and a medium $j$ illuminated from medium $i$ at the angle $\theta_{i}$ has a reflectance noted $R_{i j}\left(\theta_{i}\right)$. When illuminated from medium $j$ at the angle $\theta_{j}$, its reflectance is denoted $R_{j i}\left(\theta_{j}\right)$. An interfaced layer (respectively, interfaced multilayer) illuminated from medium $i$ at the angle $\theta_{i}$ has a reflectance noted $R_{i j k}\left(\theta_{i}\right)$ (respectively, $R_{i j k l . . .}\left(\theta_{i}\right)$, where the labeling numbers $i, j, k \ldots$ are arranged in the same order as their corresponding media, starting from the surrounding medium of incidence. The same notations are used for the transmittance.

\section{Geometrical Optics}

Within a nonscattering layer, light is attenuated according to Beer's law [8]. When light is oriented perpendicularly to the layer, its attenuation factor $t$ is called "normal transmittance." When it follows another direction, its path across the layer is longer and its attenuation is stronger. Thus, light oriented by an angle $\theta$ travels a path of relative length $1 / \cos \theta$ and is attenuated by the factor $t^{1 / \cos \theta}$.
At a flat interface between two media of different refractive indices, the directions and the ratios of reflection and transmission of light are given, respectively, by Snell's laws and Fresnel's formulas [9]. Let $n_{i}$ and $n_{j}$ be the refractive indices of the upper and the lower surrounding media, respectively. When light is incident from the upper medium at angle $\theta_{i}$, its upper reflectance $R_{i j}\left(\theta_{i}\right)$ (also called reflectivity) and its downward transmittance $T_{i j}\left(\theta_{i}\right)$ (transmittivity) satisfy the relation

$$
T_{i j}\left(\theta_{i}\right)=1-R_{i j}\left(\theta_{i}\right) .
$$

The light transmitted into medium $j$ is refracted at the angle $\theta_{j}=\arcsin \left(n_{i} \sin \theta_{i} / n_{j}\right)$. When the interface is illuminated from medium $j$ at this angle $\theta_{j}$, its lower and upper reflectances are equal and its downward and upward transmittance are equal; that is,

$$
\begin{aligned}
& R_{j i}\left(\theta_{j}\right)=R_{i j}\left(\theta_{i}\right), \\
& T_{j i}\left(\theta_{j}\right)=T_{i j}\left(\theta_{i}\right) .
\end{aligned}
$$

\section{Interfaced Layer}

An interfaced layer is a nonscattering layer whose refractive index $n_{1}$ is different from the refractive indices of its surrounding media $\left(n_{0}\right.$ at the upper side and $n_{2}$ at the lower side). The light coming from medium 0 at an angle $\theta_{0}$ is refracted into the layer (medium 1 ), then into medium 2 at the respective angles $\theta_{1}$ and $\theta_{2}$ given by Snell's law,

$$
n_{0} \sin \theta_{0}=n_{1} \sin \theta_{1}=n_{2} \sin \theta_{2} .
$$

Between the interfaces, the light undergoes multiple reflections as shown in Fig. 1. At each travel across the layer, it is attenuated according to Beer's law with respect to the layer transmittance at normal incidence $t(\lambda)$, also called "normal transmittance." The ratios of emerging light at the upper and lower sides form geometric series whose sums express the interfaced layer's upper reflectance $R_{012}\left(\theta_{0}\right)$ and downward transmittance $T_{012}\left(\theta_{0}\right)$ :

$$
R_{012}\left(\theta_{0}\right)=R_{01}\left(\theta_{0}\right)+\frac{T_{01}^{2}\left(\theta_{0}\right) R_{12}\left(\theta_{1}\right) t^{2 / \cos \theta_{1}}}{1-R_{01}\left(\theta_{0}\right) R_{12}\left(\theta_{1}\right) t^{2 / \cos \theta_{1}}},
$$

and

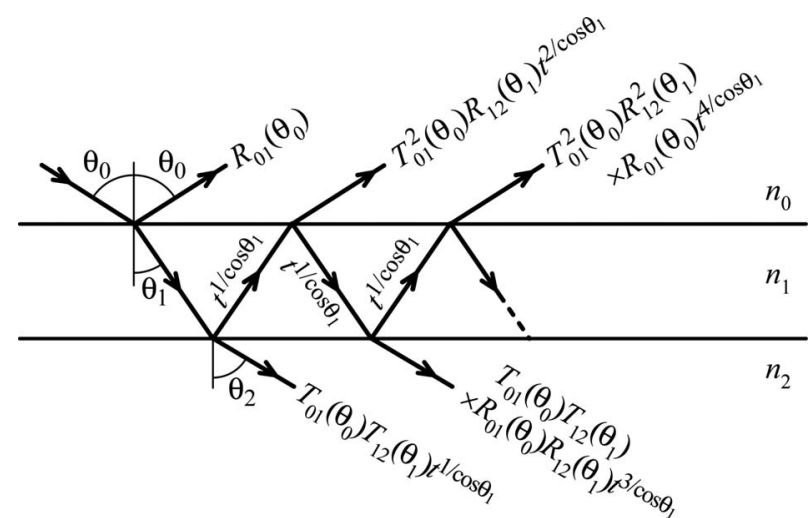

Fig. 1. Reflection and transmission of light by an interfaced layer. 


$$
T_{012}\left(\theta_{0}\right)=\frac{T_{01}\left(\theta_{0}\right) T_{12}\left(\theta_{1}\right) t^{1 / \cos \theta_{1}}}{1-R_{01}\left(\theta_{0}\right) R_{12}\left(\theta_{1}\right) t^{2 / \cos \theta_{1}}} .
$$

The lower reflectance $R_{210}\left(\theta_{2}\right)$ and the upward transmittance $T_{210}\left(\theta_{2}\right)$ have the same expressions as their upper and downward equivalents but with exchanged subscripts 0 and 2 .

\section{E. Interfaced Multilayer}

An interfaced multilayer is formed by $k$ nonscattering layers with respective refractive indices $n_{j}$ and normal transmittances $t_{j}, j=1,2, \ldots, k$. The upper and lower surrounding media have the refractive indices $n_{0}$ and $n_{k+1}$, respectively. As in the previous single interfaced layer, light is multiply reflected between the different interfaces. Because of the high number of interfaces, the multiple reflection process is tedious to describe, but a simple iterative method enables calculating the multilayers' reflectance and transmittance in successive steps. At the first step, we consider the top interface alone. At the second step, we aggregate to the top interface the first layer and the second interface. At the $j$ th step, we aggregate the $(j-1)$ th layer and the $j$ th interface. We obtain a multilayer whose upper reflectance, lower reflectance, downward transmittance, and upward transmittance are given, respectively, by

$$
\begin{aligned}
& R_{0 . . i j}\left(\theta_{0}\right)=R_{0 . . i}\left(\theta_{0}\right)+\frac{T_{0 . . i}^{2}\left(\theta_{0}\right) R_{i j}\left(\theta_{i}\right) t_{i}^{2 / \cos \theta_{i}}}{1-R_{i . .0}\left(\theta_{i}\right) R_{i j}\left(\theta_{i}\right) t_{i}^{2 / \cos \theta_{i}}}, \\
& R_{j i . .0}\left(\theta_{j}\right)=R_{j i}\left(\theta_{j}\right)+\frac{T_{i j}^{2}\left(\theta_{i}\right) R_{i . .0}\left(\theta_{i}\right) t_{i}^{2 / \cos \theta_{i}}}{1-R_{i . .0}\left(\theta_{i}\right) R_{i j}\left(\theta_{i}\right) t_{i}^{2 / \cos \theta_{i}}}, \\
& T_{0 . . i j}\left(\theta_{0}\right)=\frac{T_{i j}\left(\theta_{i}\right) T_{0 . . i}\left(\theta_{0}\right) t_{i}^{1 / \cos \theta_{i}}}{1-R_{i . .0}\left(\theta_{i}\right) R_{i j}\left(\theta_{i}\right) t_{i}^{2 / \cos \theta_{i}}}, \\
& T_{j i . .0}\left(\theta_{j}\right)=\frac{T_{i j}\left(\theta_{i}\right) T_{i . .0}\left(\theta_{i}\right) t_{i}^{1 / \cos \theta_{i}}}{1-R_{i . .0}\left(\theta_{i}\right) R_{i j}\left(\theta_{i}\right) t_{i}^{2 / \cos \theta_{i}}},
\end{aligned}
$$

with $i=j-1$. When $j=k+1$, the reflectances and the transmittances of the complete multilayer are obtained.

Note that at step 1, as a property of the Fresnel transmittivity of the top interface, the downward and upward transmittances are equal, i.e., $T_{01}\left(\theta_{0}\right)=T_{10}\left(\theta_{1}\right)$. It follows from Eqs. (8) and (9) that the downward and upward transmittances are also equal at each step, i.e., $T_{0 . . i j}\left(\theta_{0}\right)$ $=T_{j i . .0}\left(\theta_{j}\right)$. The upper and lower reflectances are equal only when the interfaced multilayer is symmetric, i.e., identical to itself when reversed (upside-down).

\section{F. Total Reflection}

The transmittance of an interfaced multilayer becomes zero when the light is totally reflected at an interface. The interface where the total reflection occurs is the first interface encountered by light whose refractive index is inferior to $n_{0} \sin \theta_{0}, \theta_{0}$ being the angle of incidence. In the example illustrated in Fig. 2, a multilayer is illuminated from the bottom side at different angles $\theta_{3}$. The light ray

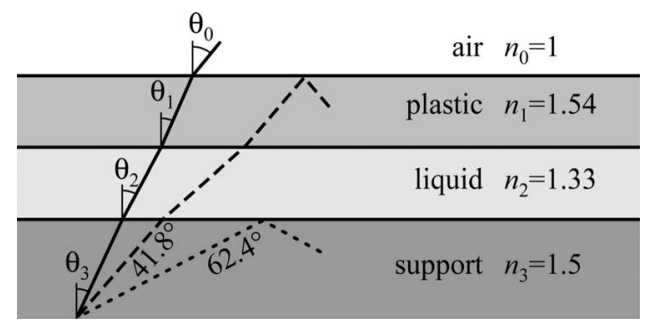

Fig. 2. Transmission and total reflection of light rays within a multilayer.

can cross the multilayer as long as $n_{3} \sin \theta_{3}$ is superior to each of the multilayer refractive indices $n_{2}, n_{1}$ and $n_{0}$, that is, in the present case, as long as $\theta_{3}$ is inferior to $41.8^{\circ}=\arcsin (1 / 1.5)$. For larger angles $\theta_{3}, n_{3} \sin \theta_{3}$ becomes first superior to $n_{0}$, then superior to $n_{2}$, yielding consequently a total reflection at, respectively, the plastic-air interface and the support-liquid interface.

However, even in the case where total reflection occurs, the iterative reflectance and transmittance calculation can be performed from $j=1$ to $j=k+1$, and the effect of total reflection is automatically taken into account. This can be shown easily by recalling that the reflectivity is 1 at the interface where the total reflection occurs, that is,

$$
R_{i j}\left(\theta_{i}\right)=\left\{\begin{array}{ll}
1 & n_{j} \leqslant n_{0} \sin \theta_{0} \\
R_{i j}\left(\theta_{i}\right) & n_{j}>n_{0} \sin \theta_{0}
\end{array},\right.
$$

and that the transmittivity is zero. Consequently, transmittance $T_{0 . . i j}\left(\theta_{0}\right)$ given by Eq. (8) is also zero. In the next iterations, the reflectance remains unchanged and the transmittance remains equal to zero.

\section{G. Lambertian Illumination}

When the incident light is Lambertian, the interfaced multilayer receives a collection of light rays following their own directional path according to their initial direction. The "diffuse reflectance" and the "diffuse transmittance" account for the reflection (respectively, the transmission) of all these rays and are expressed by an integral of the form [7]

$$
f=\int_{\theta=0}^{\pi / 2} F(\theta) \sin 2 \theta \mathrm{d} \theta,
$$

where $F$ is the reflectance or the transmittance of the interfaced multilayer as a function of the incident angle $\theta$.

Note that a certain fraction of incident light is subject to total reflection within the interfaced multilayer, depending on the refractive index of the medium of incidence. Thus, when the upper and the lower surrounding media have different refractive indices, the upward and downward diffuse transmittances are different. Only a symmetric interfaced multilayer has identical downward and upward diffuse transmittances and identical upper and lower diffuse reflectances.

\section{H. Radiance Detector}

An interfaced multilayer is observed by a radiance detector at angle $\theta_{0}$ in medium 0 (refractive index $n_{0}$ ) and illuminated by Lambertian light in medium $k$ (refractive 
index $n_{k}$ ). The radiance $L_{t}$ captured by the detector is $[10,11]$

$$
L_{t}=\frac{\mathrm{d}^{2} \Phi_{t}}{\mathrm{~d} s \cos \theta_{0} \sin \theta_{0} \mathrm{~d} \theta_{0} \mathrm{~d} \phi},
$$

where $\mathrm{d}^{2} \Phi_{t}$ is the captured flux element, $\mathrm{d} s$ denotes an infinitesimal area of the multilayer, and $\phi$ is the azimuth angle. Since the interfaced multilayer is nonscattering, the part of incident light that crosses it in the direction of the detector is a light pencil defined by the radiance

$$
L_{i}=\frac{\mathrm{d}^{2} \Phi_{i}}{\mathrm{~d} s \cos \theta_{k} \sin \theta_{k} \mathrm{~d} \theta_{k} \mathrm{~d} \phi},
$$

where $\mathrm{d}^{2} \Phi_{i}$ is the corresponding incident flux element and angle $\theta_{k}$ denotes the pencil orientation in medium $k$. When crossing the interfaced multilayer, the incident flux element $\mathrm{d}^{2} \Phi_{i}$ is attenuated by the multilayer transmittance $T_{k . .0}\left(\theta_{k}\right)$, also equal to $T_{0 . . k}\left(\theta_{0}\right)$. The captured flux element is therefore

$$
\mathrm{d}^{2} \Phi_{t}=T_{0 . . k}\left(\theta_{0}\right) \mathrm{d}^{2} \Phi_{i} .
$$

According to Snell's laws, angles $\theta_{0}$ and $\theta_{k}$ satisfy the relation

$$
\sin \theta_{0}=\left(n_{k} / n_{0}\right) \sin \theta_{k} .
$$

By differentiating both members of Eq. (15), we obtain

$$
\cos \theta_{0} \mathrm{~d} \theta_{0}=\left(n_{k} / n_{0}\right) \cos \theta_{k} \mathrm{~d} \theta_{k},
$$

and by replacing in Eq. (12), respectively, $\mathrm{d}^{2} \Phi_{t}$, $\sin \theta_{0}$, and $\cos \theta_{0} \mathrm{~d} \theta_{0}$ according to Eqs. (14)-(16), we obtain the relation between the incident and captured radiances:

$$
L_{t}=\left(n_{0} / n_{k}\right)^{2} T_{0 . . k}\left(\theta_{0}\right) L_{i}
$$

The factor $\left(n_{0} / n_{k}\right)^{2}$, characteristic of the change of solid angle due to the refraction, is independent of the detector orientation.

\section{Polarization}

The reflectance and transmittance of the multilayer depends on the polarization of light. In our model, we consider incoherent and unpolarized light, which is classically modeled as the junction of two independent light sources with linear polarization [[9] pp. 45-46]. The two polarized components follow the same path within the multilayer and yield identical reflectance and transmittance expressions, but with different Fresnel coefficients. After computing each of the polarized reflectances and transmittances, they are averaged to obtain the reflectance and transmittance for natural light. Note that a diffusing support such as the one considered in Section 6 cancels the polarization of light due to intense incoherent scattering. Thus, when reflecting light, the diffusing support averages the incident polarization components and re-emits unpolarized light in the same manner as a natural light emitter.

\section{CHARACTERIZATION OF A NONSCATTERING SHEET}

A nonscattering sheet is a layer of nonscattering medium bordered by flat surfaces. According to the model presented in Section 2, the sheet is an interfaced layer characterized by its refractive index and its normal transmittance. Let us show how these two parameters can be deduced from measurements.

The sheet reflectance and the sheet transmittance are measured at normal incidence using, for example, a Lambertian light and a radiance detector at $0^{\circ}$. The relation between the measured reflectance $R(\lambda)$, the normal transmittance, and the refractive index is given by Eq. (4), where subscript 1 denotes the sheet medium, subscripts 0 and 2 denote both the surrounding air, and where $\theta_{0}=\theta_{1}$ $=0$. Let us use for the Fresnel coefficients the notations $r_{0}=R_{10}(0)=R_{01}(0)=R_{12}(0)=R_{21}(0) \quad$ and $\quad T_{01}(0)=T_{12}(0)$ $=1-r_{0}$. Equation (4) becomes

$$
R(\lambda)=r_{0}+\frac{\left(1-r_{0}^{2}\right) r_{0} t^{2}(\lambda)}{1-r_{0}^{2} t^{2}(\lambda)}
$$

We deduce from Eq. (18) an explicit expression for the layer's normal transmittance $t(\lambda)$ :

$$
t(\lambda)=\sqrt{\frac{R(\lambda)-r_{0}}{r_{0}\left[1-2 r_{0}^{2}+r_{0} R(\lambda)\right]}} .
$$

Equation (5), with the same notations as above, gives the following expression for the measured transmittance $T(\lambda)$ :

$$
T(\lambda)=\frac{\left(1-r_{0}\right)^{2} t(\lambda)}{1-r_{0}^{2} t^{2}(\lambda)} .
$$

We deduce from Eq. (20) a second explicit expression for $t(\lambda)$ :

$$
t(\lambda)=\frac{\sqrt{\left(1-r_{0}\right)^{4}+4 r_{0}^{2} T^{2}(\lambda)}-\left(1-r_{0}\right)^{2}}{2 r_{0}^{2} T(\lambda)} .
$$

In practice, formula (21) is more accurate than formula (19) for three reasons. First, the reflectance of a nonscattering sheet is low (about $\approx 4-5 \%$ ), and the precision of its measurement depends on the sensitivity of the detector. The transmittance is much higher and therefore more appropriate for a reliable measurement. Second, the reflection of light by the nonscattering sheet is due only to the interfaces, which may present small heterogeneities. These defects affect noticeably the reflectance, whereas they are irrelevant in transmission. Third, the value of $r_{0}$, and therefore the value of the sheet's refractive index $n_{1}$, has an important influence on the result returned by formula (19), whereas it has almost no influence on the result returned by formula (21). Therefore, we recommend computing $t(\lambda)$ from the measured transmittance $T(\lambda)$ by the use of Eq. (21).

Nevertheless, we can take benefit of the dependence of formula (19) on the refractive index $n_{1}$ in order to estimate it accurately. A precise value of $n_{1}$ will be needed when modeling the reflection and the transmission of a pile of sheets. When the value of $n_{1}$ corresponds precisely 
to the refractive index of the sheet, the deviation between the transmission spectra deduced from formulas (19) and (21) is minimal. Thus, $n_{1}$ can be fitted so as to minimize the average squared difference between the two deduced spectra.

\section{PILES OF NONSCATTERING SHEETS}

Identical sheets of refractive index $n_{1}$ and normal transmittance $t(\lambda)$ are superposed one on top of another (Fig. 3). Between the sheets, a binding layer is formed by a nonscattering and nonabsorbing medium of refractive index $n_{2}$, with normal transmittance equal to 1 . The top and bottom sheets are bordered by air $\left(n_{0}=1\right)$. The specimen obtained is an interfaced multilayer whose upper reflectance and downward transmittance are calculated iteratively with formulas (6)-(9). They are noted respectively $R_{012 \ldots 10}\left(\theta_{0}\right)$ and $T_{012 \ldots 10}\left(\theta_{0}\right)$, where the indices indicate the sequence of the different media. Since the pile considered is symmetric, its lower reflectance and its upward transmittance are equal, respectively, to its upper reflectance and its downward transmittance.

\section{A. Experimental Verification}

The model was verified with nonscattering sheets of blue plastic whose refractive index $n_{1}=1.54$ was fitted according to the method proposed in Section 3 and whose normal transmittance $t(\lambda)$ was calculated from Eq. (21). When the sheets are simply laid on top of one another, they are naturally separated by a thin layer of air $\left(n_{2}\right.$ $=1$ ) and form an "air-bound pile." We obtain "liquid-bound piles" by depositing a drop of liquid between each sheet. The liquid spreads out and forms a uniform layer covering the whole area of the sample. The liquid is a clear alcohol with a fitted refractive index $n_{2}=1.33$. All the measurements were performed at normal incidence $\left(\theta_{0}=0^{\circ}\right)$.

Measurements and predictions concern the reflectance of air-bound piles (Fig. 4), the transmittance of air-bound piles (Fig. 5), and the transmittance of liquid-bound piles (Fig. 6). For each pair of predicted and measured spectra, the circled number indicates the number of sheets, and the CIELAB $\Delta \mathrm{E}_{94}$ value represents the colorimetric difference between these two spectra, with a $D_{65}$ illuminant and a surrounding perfectly reflective or transmissive white field [12]. Note the different scales that are used for the reflectance plots and the transmittance plots. The very low $\Delta \mathrm{E}_{94}$ values obtained in transmittance mode demonstrate the excellent accuracy of the transmittance predictions (Figs. 5 and 6). In reflectance mode (Fig. 4), the small $\Delta \mathrm{E}_{94}$ values validate the prediction model despite being slightly larger than in transmittance mode.

\begin{tabular}{ll}
$n_{0}$ & air \\
\hline$n_{1}$ & nonscattering sheet \\
\hline$n_{2}$ & binding layer \\
\hline$n_{1}$ & nonscattering sheet \\
\hline$n_{2}$ & binding layer \\
\hline$n_{1}$ & nonscattering sheet \\
\hline$n_{0}$ & air
\end{tabular}

Fig. 3. Pile composed of three nonscattering sheets.

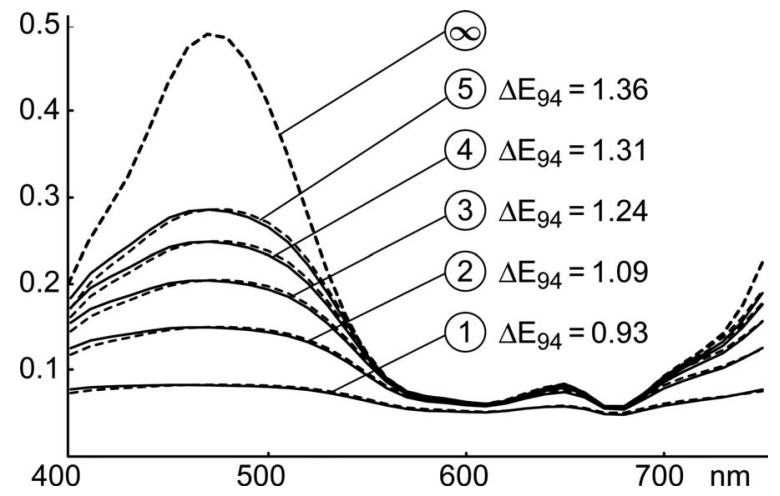

Fig. 4. Measured (solid curves) and predicted (dashed curves) reflectance spectra of air-bound piles of blue plastic ( 1 to 5 sheets, and infinity of sheets).

\section{B. Plastic-Bound Piles}

In the special case where the binder has the same refractive index as the plastic sheets $\left(n_{2}=n_{1}=1.54\right)$, the sheetbinder interfaces have no optical effect. The corresponding piles, called "plastic-bound piles," are equivalent to a simple interfaced layer with normal transmittance $t^{k}$, where $t$ is the normal transmittance of a single sheet and $k$ the number of sheets. Its global reflectance and transmittance are given by Eq. (18), [respectively, by Eq. (20), with $t$ being replaced by $t^{k}$ ]. The plastic-bound piles have nearly the same transmittance spectrum as the liquidbound piles (Fig. 6). The reflectance spectra for plasticbound piles comprising 1 to 5 superposed sheets are plotted in Fig. 7.

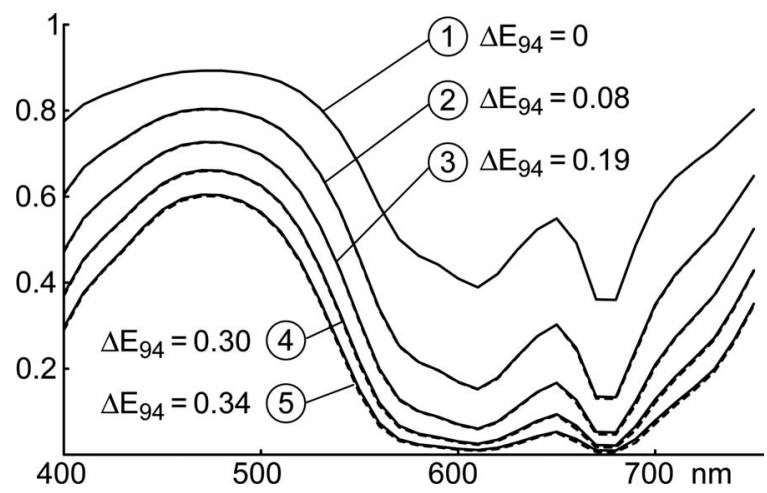

Fig. 5. Measured (solid curves) and predicted (dashed curves) transmittance spectra of air-bound piles of blue plastic sheets (1 to 5 sheets).

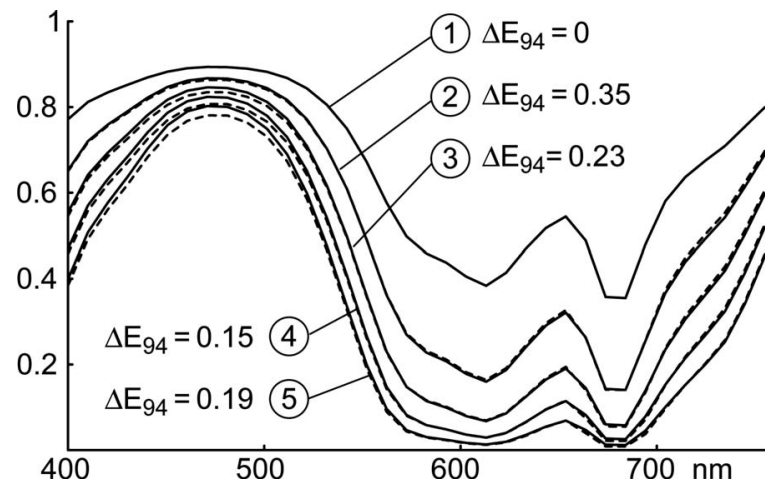

Fig. 6. Measured (solid curves) and predicted (dashed curves) transmittance spectra of liquid-bound piles of blue plastic sheets (1 to 5 sheets). 


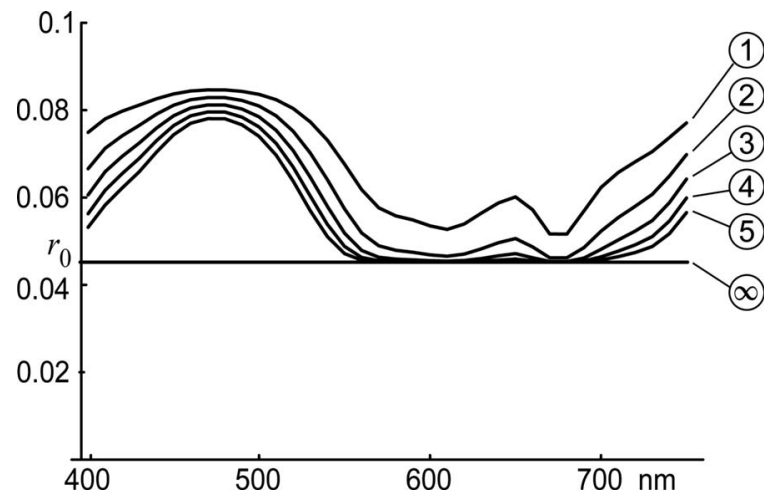

Fig. 7. Reflectance of plastic-bound piles of blue plastic sheets.

\section{Spectrum and Color Variations}

The interaction between light and piles may be summarized as the combination of an absorption phenomenon within the layers and of a back-reflection phenomenon at the interfaces. The absorption phenomenon, represented in our model by the normal transmittance spectrum $t(\lambda)$, attenuates both the reflectance and the transmittance as a function of wavelength. The back-reflection phenomenon tends to increase the reflectance and to decrease the transmittance in a proportion that depends on the reflectivity of the interfaces, therefore on the refractive index of the binder but not on the wavelength. The two phenomena are amplified when one sheet is added to the pile. This yields spectral variations depending on the wavelength and on the refractive index of the binder.

The spectra plotted in Figs. 4-7 give an idea of how reflectance and transmittance vary in the case of blue plastic sheets. To study the corresponding color variations, we predict a large set of spectra by considering piles of 2 to 200 blue plastic sheets where the binder is either air, liquid $\left(n_{2}=1.33\right)$, or plastic $\left(n_{2}=n_{1}=1.54\right)$. These spectra are converted into colors in the CIELAB color space [12]. Colors are expressed by the three coordinates $L^{*}, C^{*}$, and $h^{*}$, which correspond, respectively, to lightness, chroma, and hue. Lightness corresponds to the global elevation of the spectrum. Chroma shows the saturation of colors, i.e., the variation in spectral distribution among the different wavelength ranges. A uniform spectrum is achromatic, i.e., $C^{*}=0$. Coordinates $C^{*}$ and $h^{*}$ are derived from the classical coordinates $a^{*}$ and $b^{*}$ according to the formulas

$$
\begin{aligned}
& C^{*}=\sqrt{a^{*^{2}+b^{* 2}}} \\
& h^{*}=\arctan \left(b^{*} / a^{*}\right)
\end{aligned}
$$

For a convenient visualization of the colors of the considered piles, the coordinates $L^{*}, C^{*}$, and $h^{*}$ are considered by pair and presented in $\left(C^{*}, L^{*}\right)$ and $\left(C^{*}, h^{*}\right)$ diagrams in Fig. 8. The sequence of points obtained when incrementing the number of sheets are joined and form a curve for each type of binder. The numbers indicate the corresponding number of superposed sheets; the symbol $\infty$ indicates 200 superposed sheets.

Let us describe first the variations in transmission mode. When a sheet is added to the pile, the light crosses one additional plastic layer and two additional interfaces. The resulting attenuation is increased because of both absorption and back-reflection. The transmittance spectrum decreases (see Figs. 5 and 6), as well as the lightness of the transmitted color. The attenuation due to absorption involves mainly the wavelength belonging to the plastic absorption range $(570-680 \mathrm{~nm})$. When incrementing the number of sheets, the transmittance strongly decreases in this range and trends rapidly toward zero. Outside this range, the transmittance decreases more slowly, in proportion to the reflectivity of the added sheet-binder interfaces.

The different spectrum variations inside and outside the plastic absorption range explain the evolution of the chroma. When the spectrum varies strongly in the absorption range, the difference between high and low spec-
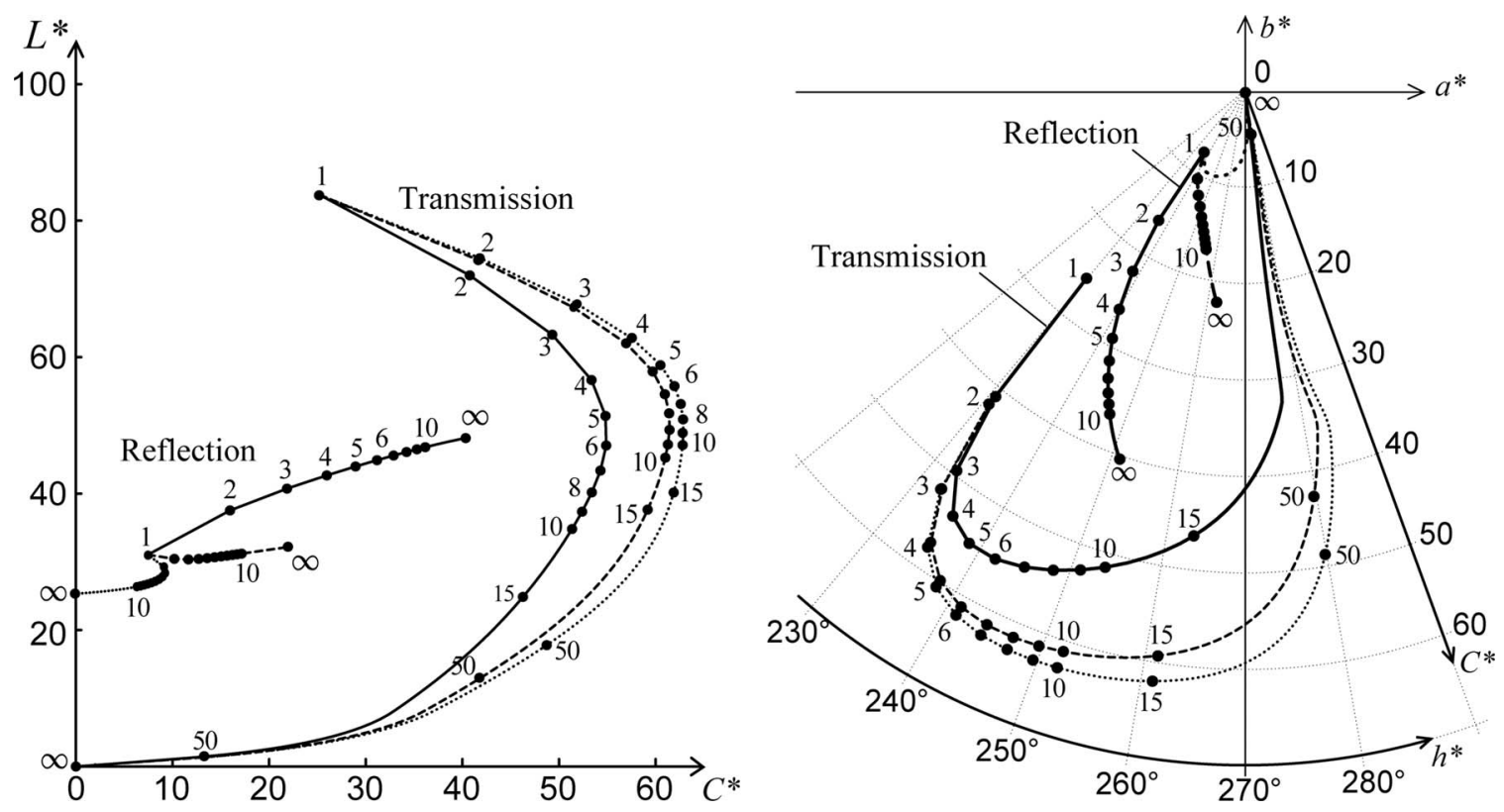

Fig. 8. $\left(L^{*}, C^{*}\right)$ coordinates (left) and $\left(C^{*}, h^{*}\right)$ coordinates (right) of the colors reflected and transmitted by air-bound piles (solid curves), liquid-bound piles (dashed curves) and plastic-bound piles (dotted curves) as a function of the number of sheets. 
trum values widens, thereby increasing the color saturation. Once the spectrum is zero in the absorption range, i.e., beyond $6-8$ sheets, adding a sheet attenuates the rest of the spectrum and reduces the difference between the highest and the lowest spectrum values, thereby decreasing the saturation. To obtain a pile with the highest chroma in transmission, one may compute the optimal number of sheets that maximizes the absorption phenomenon owing to a sufficient number of plastic layers, and at the same time minimizes the back-reflection phenomenon owing to a minimal number of interfaces. For maximal chroma, the binder-sheet interfaces should reflect as little light as possible, i.e., the refractive indices of the binder and the sheets should be as close as possible.

Regarding the hue, it undergoes a substantial variation when the pile grows and converges toward the hue of the less attenuated wavelengths (narrow band around $475 \mathrm{~nm}$ ). Since absorption is the only phenomenon responsible for chromatic variations, it is natural to observe that the binder has no effect on the hue: The three points relative to a same number of sheets are located nearly at the same angle, i.e., the three types of pile have the same hue.

Let us now study the spectral and color variations in reflection mode. In a pile, light is reflected only by the interfaces. The two external air-sheet interfaces have a fixed reflectivity equal to $r_{0}=0.045$ at normal incidence in the case of the blue plastic. The pile reflectance cannot be below this value. The other interfaces, i.e., the sheetbinder interfaces, are more or less reflecting according to the binder's refractive index. Part of the reflected light is instantaneously absorbed within the plastic layers, especially in the absorption wavelength range-in the present case between 570 and $680 \mathrm{~nm}$. When the binder is air, the binder-sheet interfaces have the highest reflectivity. Increasing the number of sheets increases the reflectance spectrum thanks to the added interfaces, but only outside the absorption range. This explains why the reflected color increases both in lightness and in chroma. As the pile grows to infinity, the reflectance converges to a maximum $R_{\infty}(\lambda)$, plotted in Fig. 4 . The reflectance spectrum and the reflected color follow the same evolution when the binder is liquid, but to a lesser extent since the sheetbinder interfaces have a lower reflectivity. When the binder has the same refractive index as the sheets (Fig. 7 ), the variation of reflectance is radically different since the sheet-binder interfaces have no optical effect. Light is reflected only at the top and the bottom interfaces. The light reflected by the bottom interface is strongly attenuated by the plastic layers. As the pile becomes infinite, the pile reflectance is reduced to the achromatic reflectivity $r_{0}=0.045$ of the top interface alone. The chroma decreases toward zero. Finally, we note that hue variations are weaker in reflection mode than in transmission mode.

\section{SHEET COMPOSITION MODEL FOR AIR-BOUND PILES}

When the nonscattering sheets are separated by air, the prediction model presented in Section 4 can be reduced to simpler equations because all the interfaces have the same reflectivity. Every sheet in the pile corresponds to the same interfaced layer. Instead of describing the multiple reflections of light between layers and interfaces, we can describe them between these interfaced layers. The interfaced layers have the reflectance $R_{010}\left(\theta_{0}\right)$ and transmittance $T_{010}\left(\theta_{0}\right)$ of a single sheet and can be measured directly. For the sake of brevity, we note them, respectively, $R(\theta)$ and $T(\theta)$, where $\theta$ is the angle of incidence in air. We also note $R_{k}(\theta)$ and $T_{k}(\theta)$ the reflectance (respectively, the transmittance) of a pile comprising $k$ sheets. Since air-bound piles are symmetric, their reflectance and transmittance do not depend on the side of illumination.

The multiple reflections taking place between two sheets are represented in Fig. 9. At each reflection or transmission, the light strikes one of the two sheets at the same angle $\theta$. The exiting components at the upper side form a geometric series, yielding for the pile reflectance the following expression:

$$
R_{2}(\theta)=R(\theta)+\frac{T^{2}(\theta) R(\theta)}{1-R^{2}(\theta)} .
$$

Likewise, the exiting components at the lower side form a geometric series expressing the pile transmittance:

$$
T_{2}(\theta)=\frac{T^{2}(\theta)}{1-R^{2}(\theta)} .
$$

For $k$ superposed sheets, a similar multiple reflection process can be described between the upper sheet and the $k-1$ other sheets, yielding the following expression for the reflectance of the pile:

$$
R_{k}(\theta)=R(\theta)+\frac{T^{2}(\theta) R_{k-1}(\theta)}{1-R(\theta) R_{k-1}(\theta)},
$$

and, for its transmittance,

$$
T_{k}(\theta)=\frac{T(\theta) T_{k-1}(\theta)}{1-R(\theta) R_{k-1}(\theta)},
$$

where $R_{k-1}(\theta)$ and $T_{k-1}(\theta)$ are, respectively, the reflectance and the transmittance of a pile of $k-1$ sheets, themselves given by Eqs. (24) and (25) with $k$ replaced by $k-1$, and so on.

\section{A. Infinite Pile}

When the number of sheets rises to infinity, the pile forms a specular reflector whose transmittance is zero and

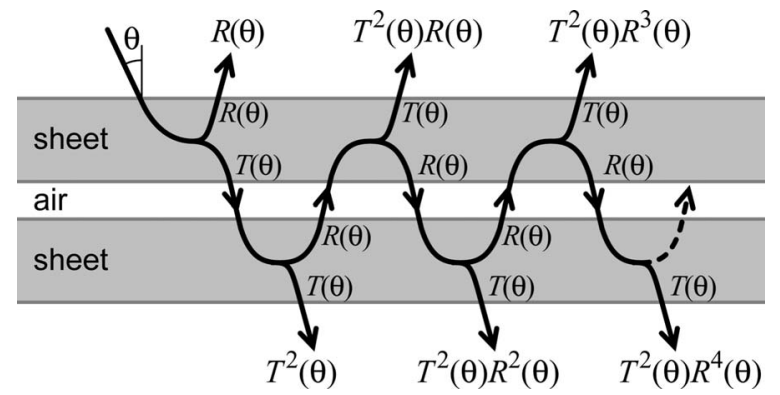

Fig. 9. Multiple reflections and transmissions of light between two identical nonscattering sheets, where the global reflectance $\mathrm{R}(\theta)$ and the global transmittance $T(\theta)$ compose the multiple reflections within each sheet. 
whose reflectance $R_{\infty}(\theta)$, plotted in Fig. 4, remains unchanged when a further sheet is added. Thus, Eq. (24) becomes:

$$
R_{\infty}(\theta)=R(\theta)+\frac{T^{2}(\theta) R_{\infty}(\theta)}{1-R(\theta) R_{\infty}(\theta)},
$$

which yields the quadratic equation

$$
R_{\infty}^{2}(\theta)-\frac{1+R^{2}(\theta)-T^{2}(\theta)}{R(\theta)} R_{\infty}(\theta)+1=0 .
$$

Equation (27) has for the infinite reflectance a single solution inferior to 1 that we may write as

$$
R_{\infty}(\theta)=a-b,
$$

with

$$
a=\frac{1+R^{2}(\theta)-T^{2}(\theta)}{2 R(\theta)}
$$

and

$$
b=\sqrt{a^{2}-1} .
$$

Equation (28) presents a striking similarity to the reflectance of infinitely thick diffusing layers expressed according to the Kubelka-Munk model [2], with only a different defining expression for $a$. This similarity is explained by the fact that both the Kubelka-Munk model and our model describe the multiple reflectiontransmission of light within a specimen whose optical properties are independent of depth [13]. In our model, light is reflected and transmitted by nonscattering sheets. In the Kubelka-Munk model, light is reflected (i.e., backscattered) and transmitted (i.e., neither backscattered nor absorbed) by infinitesimal sublayers of diffusing medium. Note also that the sheet composition model relies on the same equations as Kubelka's layering model for superposed diffusing layers $[14,15]$.

\section{B. Advantage of the Sheet Composition Model}

The sheet composition model is mathematically equivalent to the model developed in Section 4; that is, by construction Eqs. (24) and (25) are equivalent to Eqs. (6) and (8). In practice however, the direct use of measured spectra in the prediction equations avoids having to determine the refractive index and the normal transmittance of the sheets, and thus reduces the source of error. This is especially notable in reflection mode, where the predictions performed with the sheet composition model yield $\Delta \mathrm{E}_{94}$ values below 0.25 , much lower than those obtained with the layer-interface composition model presented in Fig. 4.

\section{TRANSPARENT PILES ON A DIFFUSING BACKGROUND}

Now consider placing the piles of nonscattering sheets on top of a diffusing support. The support is a Lambertian layer topped by a surface assumed to be flat. Its reflectance includes the light reflected directly by the surface and the light multiply reflected between the surface and the Lambertian layer. When a pile of nonscattering sheets is added, the Lambertian layer is topped by an interfaced multilayer comprising the pile, a layer of binder, and the binder-support interface. The specimen reflectance includes the light reflected directly by the interfaced multilayer and the light multiply reflected between the interfaced multilayer and the Lambertian layer. The support reflectance without and with an added pile can be modeled in the same way, just by replacing the support surface by the interfaced multilayer.

\section{A. Reflectance of the Support Alone}

The diffusing support is composed of a Lambertian layer whose intrinsic reflectance $\rho(\lambda)$ is independent of the incidence angle distribution. The layer has a refractive index $n_{3}$ different from that of air $\left(n_{0}=1\right)$. Thus, light is multiply reflected between the layer and its surface. The support reflectance can be expressed as $[1,11]$

$$
R_{g}(\lambda)=r_{u}+T_{\mathrm{in}} T_{\mathrm{ex}} \frac{\rho(\lambda)}{1-r_{i} \rho(\lambda)},
$$

where $r_{u}$ denotes the ratio of light reflected by the surface in the direction of the detector (specular reflection term), $T_{\text {in }}$ the ratio of incident light transmitted though the surface (penetration term), $T_{\text {ex }}$ the ratio of light transmitted from the diffusing layer to the detector (exit term), and $r_{i}$ the diffuse reflectance of the surface at the support side (internal reflectance).

The four terms $r_{u}, T_{\mathrm{in}}, T_{\mathrm{ex}}$, and $r_{i}$ derive from the reflectivity and transmittivity of the support-air interface and can be calculated once the relative refractive index $n_{3} / n_{0}$ is known. Since the internal reflectance represents the reflection of diffuse light, it is expressed by an integral similar to Eq. (11), where the integrated term is the Fresnel reflectivity of the support-air interface [16]:

$$
r_{i}=\int_{0}^{\pi / 2} R_{30}\left(\theta_{3}\right) \sin 2 \theta_{3} \mathrm{~d} \theta_{3} .
$$

The terms $r_{u}, T_{\text {in }}, T_{\text {ex }}$ depend on the measuring geometry. Their expressions are given in Appendix A for the most frequent geometries. In the case of the diffuse $/ 0^{\circ}$ geometry, where the incident light is Lambertian and the reflected light is captured by a radiance detector at $0^{\circ}$, we have

$$
\begin{aligned}
r_{u} & =R_{03}(0), \\
T_{\text {in }} & =\int_{\theta_{0}=0}^{\pi / 2} T_{03}\left(\theta_{0}\right) \sin 2 \theta_{0} \mathrm{~d} \theta_{0}, \\
T_{\text {ex }} & =\left(n_{0} / n_{3}\right)^{2} T_{03}(0),
\end{aligned}
$$

Once the support's reflectance $R_{g}(\lambda)$ is measured and once the parameters $r_{u}, T_{\text {in }}, T_{\text {ex }}, r_{i}$ are calculated with respect to the measuring geometry, the intrinsic reflectance $\rho(\lambda)$ can be obtained according to the formula, derived from Eq. (31),

$$
\rho(\lambda)=\frac{R_{g}(\lambda)-r_{u}}{T_{\mathrm{in}} T_{\mathrm{ex}}+r_{i}\left[R_{g}(\lambda)-r_{u}\right]} .
$$




\section{B. Reflectance of the Support with Pile}

The diffusing support is now superposed with a pile of nonscattering sheets. The medium binding the sheets within the pile is also used to bind the pile to the support. We call $t(\lambda)$ the normal transmittance of the sheets and $n_{0}, n_{1}, n_{2}, n_{3}$ the refractive indices of, respectively, the surrounding medium, the sheets, the binder, and the support (Fig. 10).

The support surface, the binding layer, and the pile form together an interfaced multilayer whose upper reflectance $R_{012 . .123}\left(\theta_{0}\right)$, lower reflectance $R_{321 . .210}\left(\theta_{3}\right)$, downward transmittance $T_{012 . .123}\left(\theta_{0}\right)$, and upward transmittance $T_{312 . .120}\left(\theta_{3}\right)$ depend on the normal transmittance $t(\lambda)$ and on the sequence of the different media. They are given by Eqs. (6)-(9), knowing that the binding medium has a normal transmittance equal to 1 , in the form

$$
\begin{aligned}
& R_{012 . .123}\left(\theta_{0}\right)=R_{012 . .12}\left(\theta_{0}\right)+\frac{T_{012 . .12}^{2}\left(\theta_{0}\right) R_{23}\left(\theta_{2}\right)}{1-R_{21 . .210}\left(\theta_{2}\right) R_{23}\left(\theta_{2}\right)}, \\
& R_{321 . .210}\left(\theta_{3}\right)=R_{32}\left(\theta_{3}\right)+\frac{T_{23}^{2}\left(\theta_{2}\right) R_{21 . .210}\left(\theta_{2}\right)}{1-R_{21 . .210}\left(\theta_{2}\right) R_{23}\left(\theta_{2}\right)} \\
& T_{012 . .123}\left(\theta_{0}\right)=\frac{T_{012 . .12}\left(\theta_{0}\right) T_{23}\left(\theta_{2}\right)}{1-R_{21 . .210}\left(\theta_{2}\right) R_{23}\left(\theta_{2}\right)} \\
& T_{321 . .210}\left(\theta_{3}\right)=\frac{T_{21 . .210}\left(\theta_{2}\right) T_{23}\left(\theta_{2}\right)}{1-R_{21 . .210}\left(\theta_{2}\right) R_{23}\left(\theta_{2}\right)}
\end{aligned}
$$

where $R_{012 . .12}\left(\theta_{0}\right), R_{21 . .210}\left(\theta_{2}\right), T_{012 . .12}\left(\theta_{0}\right)$, and $T_{21 . .210}\left(\theta_{2}\right)$, respectively the upper and lower reflectances and the downward and upward transmittances of the pile, are themselves obtained using iteratively Eqs. (6)-(9), as noted.

The multiple reflection process of light between this interfaced multilayer and the diffusing support, represented in Fig. 11, is similar to that taking place within the support alone. Thus, the expression for the specimen reflectance is similar to Eq. (31):

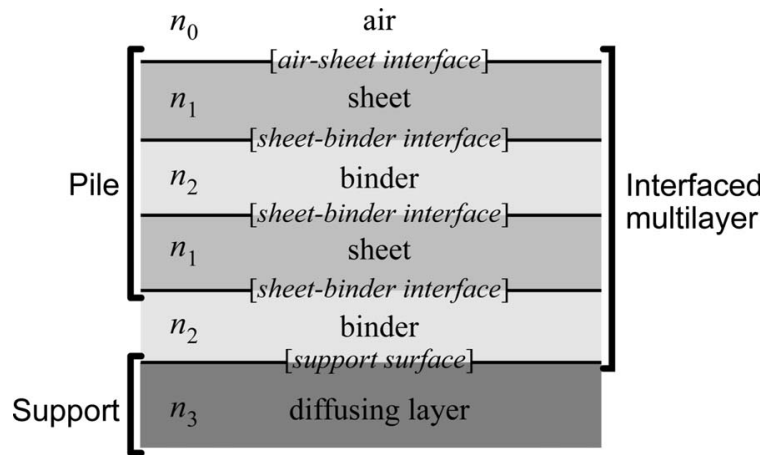

Fig. 10. Diffusing support superposed with a pile of colored nonscattering sheets.

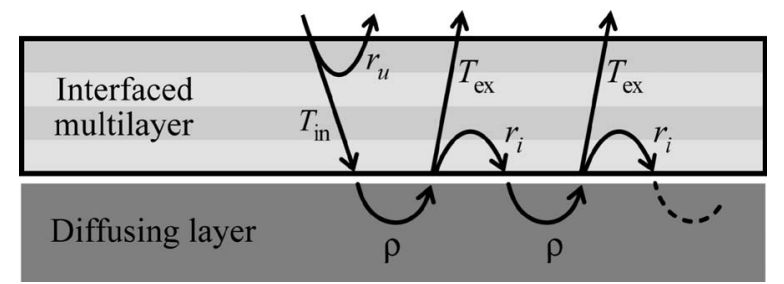

Fig. 11. Multiple reflections between the diffusing support and the interfaced multilayer.

$$
R(\lambda)=r_{u}(\lambda)+T_{\text {in }}(\lambda) T_{\text {ex }}(\lambda) \frac{\rho(\lambda)}{1-r_{i}(\lambda) \rho(\lambda)},
$$

where $\rho(\lambda)$ is the intrinsic reflectance of the support calculated according to Eq. (34), and where the terms $r_{u}(\lambda)$, $T_{\text {in }}(\lambda), T_{\text {ex }}(\lambda)$, and $r_{i}(\lambda)$ are relative to the interfaced multilayer instead of the single support-air interface. Their dependence on the wavelength is due to the normal sheet transmittance $t(\lambda)$ embodied within the interfaced multilayer reflectances and transmittances. They also vary according to the number of sheets contained in the pile and according to the binding medium. The expression for $r_{i}(\lambda)$ is similar to Eq. (32)

$$
r_{i}(\lambda)=\int_{\theta_{3}=0}^{\pi / 2} R_{321 . .210}\left(\theta_{3}\right) \sin 2 \theta_{3} \mathrm{~d} \theta_{3},
$$

and for the diffuse $/ 0^{\circ}$ geometry, the expressions for $r_{u}(\lambda)$, $T_{\text {in }}(\lambda)$, and $T_{\text {ex }}(\lambda)$ are similar to those of Eq. (33):

$$
\begin{aligned}
r_{u}(\lambda) & =R_{012 . .123}(0) \\
T_{\text {in }}(\lambda) & =\int_{\theta_{0}=0}^{\pi / 2} T_{012 . .123}\left(\theta_{0}\right) \sin 2 \theta_{0} \mathrm{~d} \theta_{0} \\
T_{\text {ex }}(\lambda) & =\left(n_{0} / n_{3}\right)^{2} T_{012 . .123}(0)
\end{aligned}
$$

\section{Experimental Verification}

The reflectance model for piles on top of a diffusing support was tested with the blue plastic sheets used in the previous sections and with a glossy white PVC support $\left(n_{3}=1.54\right.$ typical of PVC). First, the support reflectance $R_{g}(\lambda)$ was measured according to the diffuse $/ 0^{\circ}$ geometry. The intrinsic reflectance $\rho(\lambda)$ was determined according to Eq. (34) with $r_{u}, T_{\text {in }}, T_{\text {ex }}, r_{i}$ calculated according to Eqs. (32) and (33). Then, nonscattering sheets were deposited on the support. The support and the different sheets are separated by a thin layer of air $\left(n_{2}=1\right)$. We again create "liquid-bound specimens" by depositing a drop of liquid between the impermeable white PVC support and the first sheet, as well as between each sheet. We used the same liquid $\left(n_{2}=1.33\right)$ as in Section 4 . The specimen reflectances were measured according to the diffuse $/ 0^{\circ}$ geometry. They were predicted according to Eq. (39), where the terms $r_{u}(\lambda), T_{\text {in }}(\lambda), T_{\text {ex }}(\lambda), r_{i}(\lambda)$ were calculated according to Eqs. (40) and (41). The functions $R_{012 . .123}\left(\theta_{0}\right), R_{321 . .210}\left(\theta_{3}\right)$, and $T_{012 . .123}\left(\theta_{0}\right)$ were calculated iteratively with Eqs. (6)-(9) with respect to the number of sheets, the refractive index of the sheets, the refractive index of the binder, and the normal sheet transmittance 


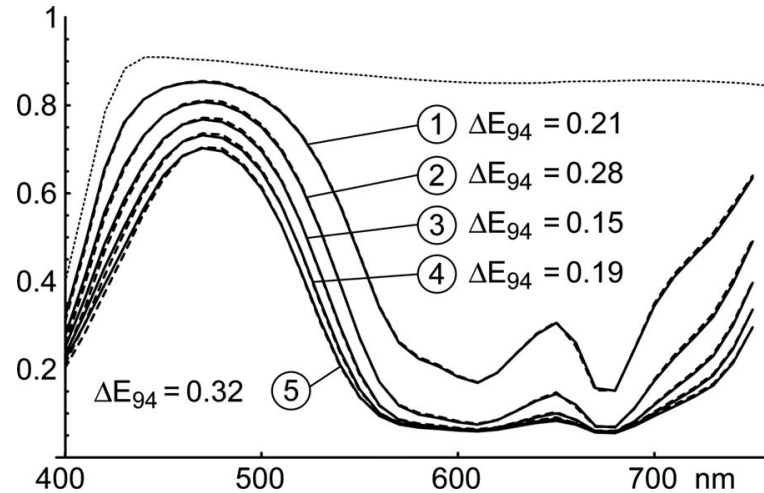

Fig. 12. Measured (solid curves) and predicted (dashed curves) reflectance spectra of blue plastic sheets superposed with a white PVC support and bound by air. Dotted curve, support reflectance spectrum.

spectrum $t(\lambda)$. The latter was deduced from the measured sheet transmittance according to Eq. (21). The same procedure was followed using a matte yellow-green paper (refractive index $n_{3}=1.5$ typical of paper) except that the liquid was not used, since it penetrates the paper and modifies its reflecting properties.

Figures 12-14 show the predicted and measured reflectance spectra of, respectively, air-bound piles superposed with the white PVC support, liquid-bound piles superposed with the white PVC support, and air-bound piles superposed with the yellow-green paper. The very low $\Delta \mathrm{E}_{94}$ values obtained in the case of the air-bound specimens (Figs. 12 and 14) demonstrates the high accuracy of the model. The predictions for the liquid-bound specimens are slightly less accurate in the short-wavelength domain (Fig. 13), possibly due to the phenomenon of dispersion by the liquid binder [9], p. 100.

\section{Case Where $n_{3}=n_{2}=n_{1}$}

In the case where the nonscattering sheets, the binder, and the diffusing support have the same refractive index, the sheet-binder interfaces have no optical effect. The sheets and the binding layers form a single nonreflecting layer bounded by the air-sheet interface at the upper side and by the diffusing layer at the lower side. We are precisely in the configuration of the Williams-Clapper model

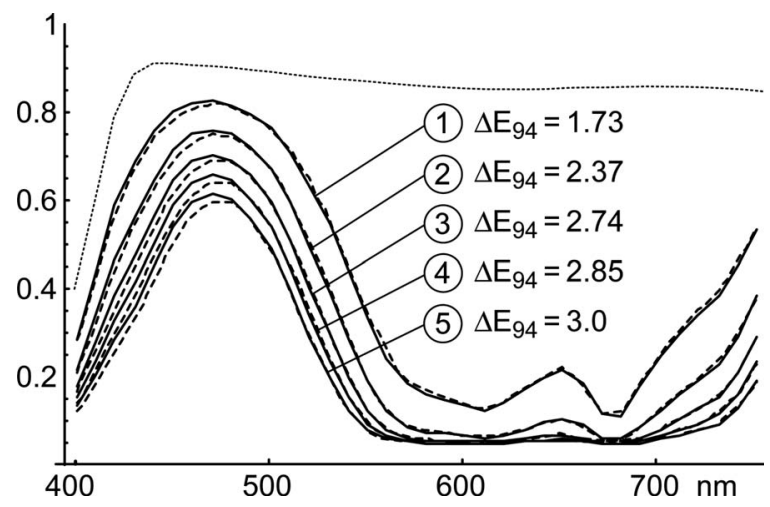

Fig. 13. Measured (solid curves) and predicted (dashed curves) reflectance spectra of blue plastic sheets superposed with a white PVC support and bound by a liquid. Dotted curve, support reflectance spectrum.

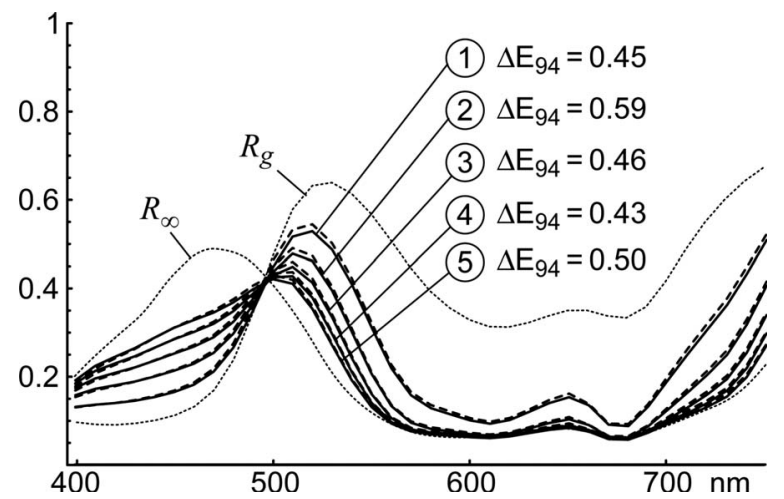

Fig. 14. Measured (solid curves) and predicted (dashed curves) reflectance spectra of blue plastic sheets superposed with a yellow-green paper support and bound by air. Dotted curve, reflectance of the support and of an infinite pile.

[3], which is a special case of our model. The nonreflecting layer has the normal transmittance $t^{k}(\lambda)$, where $t(\lambda)$ is the normal transmittance of a single sheet and $k$ is the number of sheets. Figure 15 shows the reflectance spectrum of such "plastic-bound specimens" predicted from the intrinsic reflectance of the white PVC support and the normal transmittance of the blue plastic sheets.

\section{E. Color Variations}

Let us describe the spectrum and color variations of the specimens with respect to the number of sheets, the binder's refractive index, and the support reflectance. Our study relies on specimens composed of the blue plastic sheets and the white PVC support, where the binder is either air, liquid, or plastic. The color coordinates $\left(L^{*}, C^{*}, h^{*}\right)$ of the specimens, calculated from predicted spectra for 1 to 50 plastic sheets, are represented in the $\left(C^{*}, L^{*}\right)$ and $\left(C^{*}, h^{*}\right)$ diagrams shown in Fig. 16 .

To better understand the evolution of the specimen reflectance when incrementing the number of sheets, we subdivide Eq. (39) into a first reflectance component independent of the support and represented by the term $r_{u}(\lambda)$, and a second reflectance component involving the support's intrinsic reflectance, represented by the fraction. The first component corresponds approximately to the reflectance of the pile without background and thus follows the evolution described in Section 4 when a sheet is

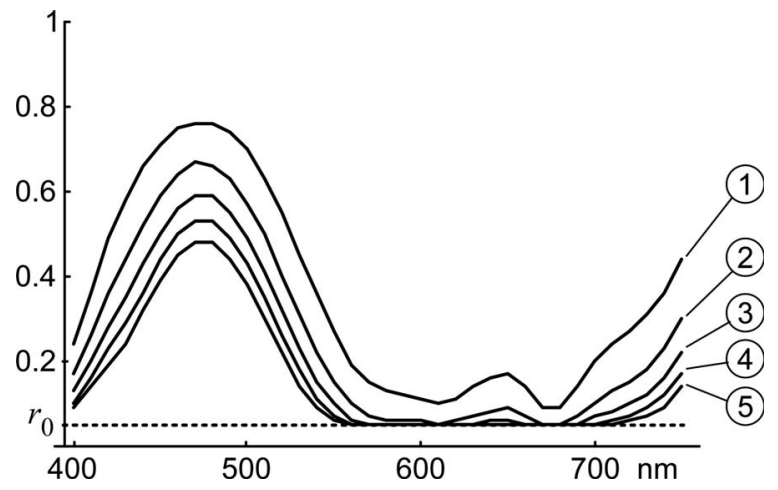

Fig. 15. Predicted reflectance spectra of plastic-bound piles of blue plastic on white PVC. 


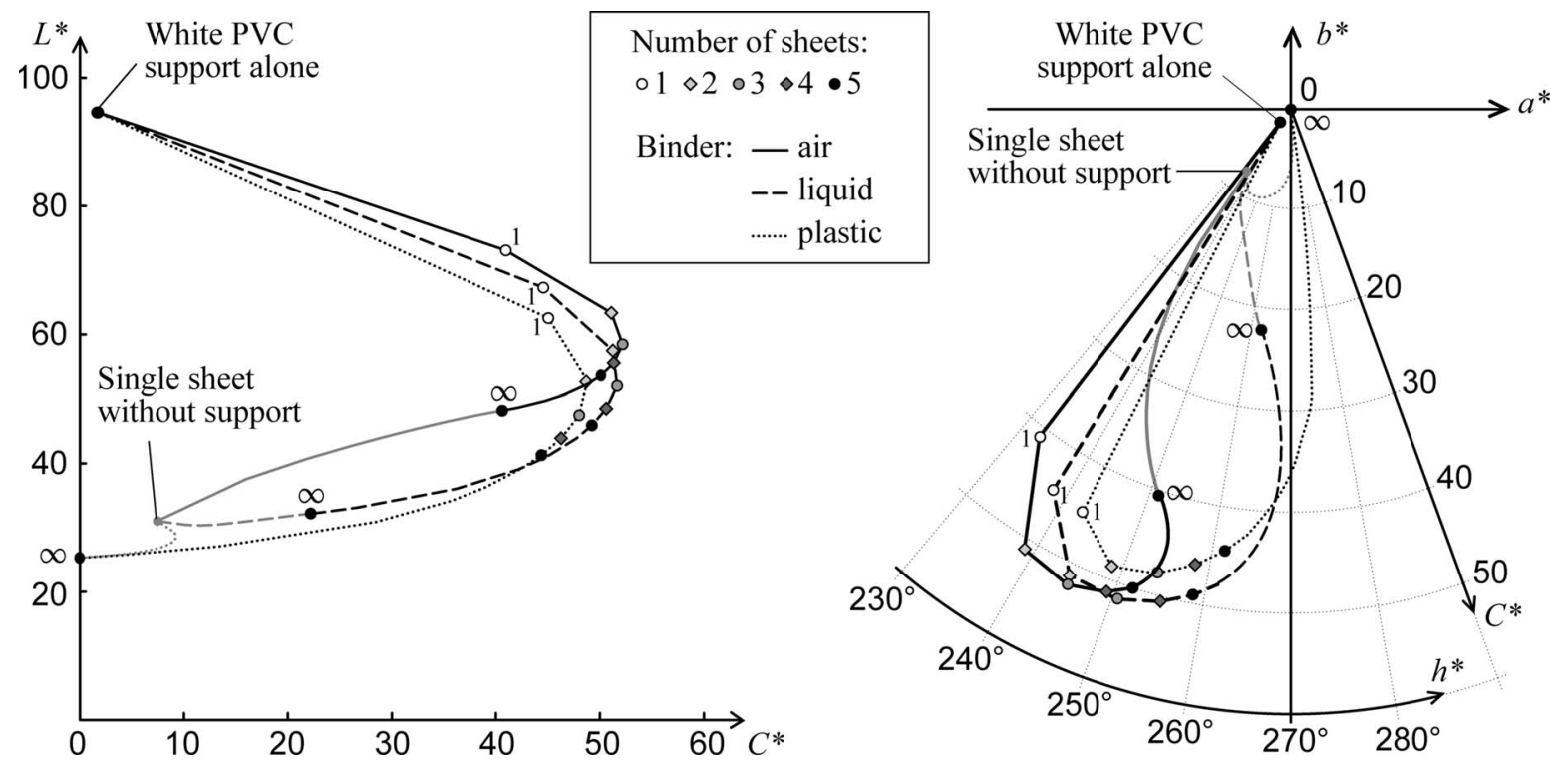

Fig. 16. $\left(C^{*}, L^{*}\right)$ coordinates (left) and $\left(C^{*}, h^{*}\right)$ coordinates of the color reflected by piles of nonscattering sheets bound by air, liquid, or plastic superposed on a white PVC support.

added. When incrementing the number of sheets, this first reflectance component increases if the binder is air or liquid, and decreases if the binder has the same refractive index as the sheets. The second reflectance component includes a transmission across the pile into the diffusing support (term $T_{\text {in }}$ ) and a transmission from the diffusing support to the air (term $T_{\mathrm{ex}}$ ). Therefore, it decreases in a similar manner as the pile transmittance when a sheet is added.

The balance between the first and the second reflectance components is determined by the support reflectance. In the case of a highly reflecting support such as the white PVC support, the second component is dominant independently of the binder. Incrementing the number of nonscattering sheets decreases the specimen reflectance (Figs. 12, 13, and 15) and decreases the lightness (Fig. 16). As in piles alone in transmission mode, the chroma reaches a maximum. This is well-known in the case of oil paintings, particularly regarding the art-glaze technique, where a white diffusing board is coated with several weakly pigmented layers $[17,18]$. The number of sheets yielding the highest chroma is approximately half the number of sheets for which the chroma of a pile alone is maximal in transmission mode. This can be explained by the fact that light reflected by the support traverses the pile twice. When the number of sheets tends to infinity, the support no longer has any influence: The pile reflectance tends to the same reflectance $R_{\infty}$ with or without background (compare the location of dots with symbol $\infty$ between Fig. 16 and Fig. 8).

When the background is colored, the balance between the first and the second components may be different for each wavelength. This appears clearly in the case of the green paper (Fig. 14), where adding a sheet increases the reflectance in the short wavelength domain and decreases the reflectance beyond $500 \mathrm{~nm}$. At the wavelength $\lambda=500 \mathrm{~nm}$, all the specimens have the same reflectance, including the support alone. We observe that this invariance phenomenon occurs precisely at the wavelength where the support reflectance $R_{g}$ coincides with the infinite air-bound pile reflectance $R_{\infty}$, which remains constant when adding sheets as explained in Section 5.

\section{CONCLUSIONS}

We have experimentally verified the accuracy of the reflectance prediction model introduced by Simonot et al. for stacked nonscattering layers on a diffusing background [7]. This model relies on the description of the multiple reflections of light between reflective and transmissive elements on the basis of the classical laws of geometrical optics (Snell's laws, Fresnel's formulas, and Beer's law), with explicit consideration of the orientation of light at each stage within the multilayer and consideration of the measuring geometry. Several extensions of the existing model are proposed in the present paper. First, we propose explicit formulas to deduce the normal sheet transmittance from the sheet reflectance or sheet transmittance measured at normal incidence. Second, we present a simple and accurate model for piles of sheets separated by air, where the reflectance and the transmittance of piles can be predicted using only the reflectance and the transmittance measured from a single sheet. We recommend using this model when the sheets are separated by air, i.e., when the binder is air. This model is valid only with directional light. It can be used neither with diffuse incident light nor in the presence of a diffusing background.

With colored plastic sheets, many specimens can be obtained easily by varying the numbers of nonscattering sheets, the binding medium, or the support. With this large set of specimens, we verified the model and studied the influence of various parameters. We showed that a maximal chroma is obtained when a certain number of colored nonscattering layers are deposited on a white background, a phenomenon well-known by painters $[17,18]$. We also showed that, when at a given wavelength the support has the same reflectance as an infinite pile of 
Table 1. Specular Reflectance of an Interface According to the Light Source and the Capturing Device

\begin{tabular}{ccc}
\hline & Radiance Detector at Angle $\theta^{\prime}$ & Integrating Sphere \\
\hline Directional incident light at angle $\theta^{\prime}$ & $r_{u}=\left\{\begin{array}{cc}R_{03}\left(\theta^{\prime}\right) & \theta^{\prime}=\theta^{\prime \prime} \\
0 & \text { otherwise }\end{array}\right.$ & $r_{u}=R_{03}\left(\theta^{\prime}\right)$ \\
Lambertian incident light & $r_{u}=R_{03}\left(\theta^{\prime}\right)$ & $r_{u}=\int_{\theta_{0}=0}^{\pi / 2} R_{03}\left(\theta_{0}\right) \sin \left(2 \theta_{0}\right) \mathrm{d} \theta_{0}$ \\
\hline
\end{tabular}

sheets, its reflectance at this wavelength is not modified when incrementing the number of sheets. The proposed model is appropriate for specimens comprising nonscattering sheets of different colors. It is also suitable for any object where a diffusing support is superposed with a nonscattering multilayer, for example, varnished surfaces, printed and laminated plastics, and transparent plastic protections.

\section{APPENDIX A: MEASURING GEOMETRIES}

In the reflectance model for diffusing support presented in Section 6, three terms depend on the measuring geometry: The specular reflection term $r_{u}$, the penetration term $T_{\text {in }}$, and the exit term $T_{\text {ex }}$. We give their expression for classical measuring devices, where the light source is either Lambertian or collimated, and where light is either captured by a radiance detector or collected by an integrating sphere. The development of these expressions relies on radiometric equations $[19,20]$. As in Section 6 , the refractive indices of the surrounding air and of the support are denoted, respectively, $n_{0}$ and $n_{3}$.

For a collimated light source, $T_{\text {in }}$ corresponds to the Fresnel transmittivity of the interface at the incident orientation angle $\theta^{\prime}$ :

$$
T_{\text {in }}=T_{03}\left(\theta^{\prime}\right) .
$$

For a Lambertian light source, $T_{\text {in }}$ accounts for all the orientations and is expressed by an integral similar to Eq. (11):

$$
T_{\text {in }}=\int_{\theta_{0}=0}^{\pi / 2} T_{03}\left(\theta_{0}\right) \sin \left(2 \theta_{0}\right) \mathrm{d} \theta_{0} .
$$

The exit term $T_{\text {ex }}$ expresses the ratio of light transmission from the background to the detector. For a radiance detector placed at angle $\theta^{\prime \prime}$, it is composed of the interface transmittivity multiplied by a factor characteristic of the variation of solid angle due to the refraction [see also Eq. (17)]:

$$
T_{\mathrm{ex}}=\left(n_{0} / n_{3}\right)^{2} T_{03}\left(\theta^{\prime}\right) .
$$

When the reflected light is collected by an integrating sphere, $T_{\text {ex }}$ accounts for the whole incident light and is expressed by an integral similar to Eq. (11):

$$
T_{\text {ex }}=\int_{\theta_{3}=0}^{\pi / 2} T_{30}\left(\theta_{3}\right) \sin \left(2 \theta_{3}\right) \mathrm{d} \theta_{3} .
$$

The specular reflection term $r_{u}$ depends both on the illumination and the capture geometries, yielding the four classical configurations presented in Table 1 . Note that some spectrophotometers exclude the specular reflection from the measurement. In this case, $r_{u}$ is zero.

\section{REFERENCES}

1. J. L. Saunderson, "Calculation of the color pigmented plastics," J. Opt. Soc. Am. 32, 727-736 (1942).

2. P. Kubelka, "New contributions to the optics of intensely light-scattering material, part I," J. Opt. Soc. Am. 38, 448-457 (1948).

3. F. C. Williams and F. R. Clapper, "Multiple internal reflections in photographic color prints," J. Opt. Soc. Am. 29, 595-599 (1953).

4. J. D. Shore and J. P. Spoonhower, "Reflection density in photographic color prints: Generalizations of the WilliamsClapper transform,” J. Imaging Sci. Technol. 45, 484-488 (2001).

5. F. R. Clapper and J. A. C. Yule, "The effect of multiple internal reflections on the densities of halftone prints on paper," J. Opt. Soc. Am. 43, 600-603 (1953).

6. M. Hébert and R. D. Hersch, "Deducing ink-transmittance spectra from reflectance and transmittance measurements of prints," Proc. SPIE 6493, 649314-1-13 (2007).

7. L. Simonot, M. Hébert, and R. D. Hersch, "Extension of the Williams-Clapper model to stacked nondiffusing colored coatings with different refractive indices," J. Opt. Soc. Am. A 23, 1432-1441 (2006).

8. H.-H. Perkampus, Encyclopedia of Spectroscopy (VCH, 1995).

9. M. Born and E. Wolf, Principles of Optics, 7th ed. (Pergamon, 1999).

10. W. R. McCluney, Introduction to Radiometry and Photometry (Artech House, 1994).

11. M. Hébert and R. D. Hersch, "Classical print reflection models: A radiometric approach," J. Imaging Sci. Technol. 48, 363-374 (2004).

12. G. Sharma, "Color fundamentals for digital imaging," in Digital Color Imaging Handbook, G. Sharma, ed. (CRC Press, 2003), pp. 30-36.

13. M. Hébert and J.-M. Becker, "Correspondence between continuous and discrete 2 flux models for reflectance and transmittance of diffusing layers," J. Opt. A, Pure Appl. Opt. 10, 035006 (2008).

14. P. Kubelka, "New contributions to the optics of intensely light-scattering materials, part II: Nonhomogeneous layers," J. Opt. Soc. Am. 44, 330-335 (1954).

15. M. Hébert, R. Hersch, and J.-M. Becker, "Compositional reflectance and transmittance model for multilayer specimens," J. Opt. Soc. Am. A 24, 2628-2644 (2007).

16. D. B. Judd, "Fresnel reflection of diffusely incident light," J. Res. Natl. Bur. Stand. 29, 329-332 (1942).

17. M. Elias and L. Simonot, "Separation between the different fluxes scattered by art glazes: Explanation of the special color saturation," Appl. Opt. 45, 3163-3172 (2006).

18. L. Simonot, M. Elias, and E. Charron, "Special visual effect of art-glazes explained by the radiative transfer equation," Appl. Opt. 43, 2580-2587 (2004).

19. M. Hébert and R. D. Hersch, "A reflectance and transmittance model for recto-verso halftone prints," J. Opt. Soc. Am. A 22, 1952-1967 (2006).

20. M. Hébert, "Compositional model for predicting multilayer reflectances and transmittances in color reproduction," Ph.D. dissertation (Ecole Polytchnique Fédérale de Lausanne, 2006), p. 139. 